\title{
Análise da Correlação Entre Independência Funcional e Satisfação com a Tecnologia Assistiva em Pessoas com Lesão Medular
}

\author{
Rogério Assunção Tannús, ${ }^{1}$ Eduarda da Rocha Ordones, ${ }^{2}$ \\ Débora Mikaelly Calaça da Silva Guerra, ${ }^{2}$ Juliane Leite Orcino, Rozany, ${ }^{2}$ \\ Cristina de Souza Melo, ${ }^{2}$ Antonio Márcio Teodoro Cordeiro Silva, ${ }^{3}$ \\ Rogério José de Almeida ${ }^{3}$
}

\section{RESUMO}

A lesão medular (LM) repercute altamente no nível de incapacidade física. Pessoas com LM que utilizam dispositivo auxiliar de mobilidade tendem a desenvolver maior independência funcional. O objetivo foi avaliar a correlação entre independência funcional em atividades de vida diária e a satisfação com o uso de tecnologia assistiva (cadeira de rodas) em pessoas com lesão medular. Trata-se de um estudo transversal analítico com abordagem quantitativa. A amostra foi de 146 participantes com lesão medular traumática com paraplegia e tetraplegia. Para coleta de dados foram utilizados um questionário sociodemográfico, as escalas Spinal Cord Independence Measure-Self Reported Version (brSCIM-SR) e o Quebec User Evaluation of Satisfaction with Assistive Technology (QUEST.2.0), que permitiram a investigação. A média de idade foi 37,8 anos, com maioria do sexo masculino (82,9\%), solteiros $(52,7 \%)$, escolaridade média $(53,4 \%)$, acidente de trânsito $(50 \%)$, arma de fogo $(22,6 \%)$. Houve predomínio de lesão completa $(58,9 \%)$ e paraplegia $(65,1 \%)$. Na brSCIM-SR a média geral foi de $55,3( \pm 15,8)$ no domínio do autocuidado de $14,6( \pm 5,3)$, respiração/esfíncter $26,4( \pm 7,4)$ e mobilidade de $14,3( \pm 5,5)$. Na satisfação com a tecnologia assistiva a pontuação total do QUEST 2.0 foi de $3,64( \pm 0,77)$, satisfação com o recurso $3,86( \pm 0,74)$ e a prestação de serviços $3,64( \pm 0,77)$. Identificou-se bom desempenho na média da independência funcional. Além disso, verificou-se altos níveis de satisfação com a tecnologia assistiva, de forma específica mais com o recurso do que com a prestação de serviços. Não foi encontrada correlação entre a independência funcional e a satisfação do uso de cadeira de rodas.

Palavras-chave: Atividades cotidianas. Cadeira de rodas. Equipamentos de Autoajuda.

\section{ANALYSIS OF THE CORRELATION BETWEEN FUNCTIONAL INDEPENDENCE AND SATISFACTION WITH ASSISTIVE TECHNOLOGY IN PEOPLE WITH SPINAL CORD INJURY}

\section{ABSTRACT}

Spinal cord injury (SCI) has a high impact on the level of physical disability. People with $\mathrm{SCl}$ who use a mobility aid device tend to develop greater functional independence. The objective was to evaluate the correlation between functional independence in activities of daily living and satisfaction with the use of assistive technology (wheelchair) in people with spinal cord injury. This is a cross-sectional analytical study with a quantitative approach. The sample consisted of 146 participants with traumatic spinal cord injury with paraplegia and quadriplegia. For data collection, a sociodemographic questionnaire was used, and the Spinal Cord Independence Measure-Self Reported Version (brSCIM-SR) and the Quebec User Evaluation of Satisfaction with Assistive Technology (QUEST.2.0) scales that allowed the investigation. The average age was 37.8 years, with a majority of males ( $82.9 \%)$, single (52.7\%), average education $(53.4 \%)$, traffic accident (50\%), firearm (22.6\%). There was a predominance of complete injury (58.9\%) and paraplegia (65.1\%). In brSCIM-SR the general mean was $55.3( \pm 15.8)$ in the self-care domain of $14.6( \pm 5.3)$, breathing/sphincter $26.4( \pm 7.4)$ and mobility of $14,3( \pm 5.5)$. In terms of satisfaction with assistive technology, the total QUEST 2.0 score was $3.64( \pm 0.77)$, satisfaction with the resource $3.86( \pm 0.74)$ and the provision of services $3.64( \pm 0.77))$. Good performance was identified in the average of functional independence. In addition, high levels of satisfaction with assistive technology, specifically more with the resource than with the provision of services. No correlation was found between functional independence and satisfaction with the use of a wheelchair.

Keyword: Activities of daily living. Spinal cord injuries. Wheelchairs.

RECEBIDO EM: 20/12/2020

MODIFICAÇÕES SOLICITADAS EM: 19/1/2021

ACEITO EM: 29/4/2021

\footnotetext{
${ }^{1}$ Autor correspondente. Universidade Estadual de Goiás - UEG. Avenida Oeste, № 250 - Setor Central. Goiânia/GO, Brasil. CEP 74575-010. http://lattes.cnpq.br/0467722540093267. https://orcid.org/0000-0002-8775-8011. rotannus@uol.com.br 2 Universidade Estadual de Goiás - UEG. Goiânia/GO, Brasil.

3 Pontifícia Universidade Católica de Goiás - PUC Goiás. Goiânia/GO, Brasil.
} 


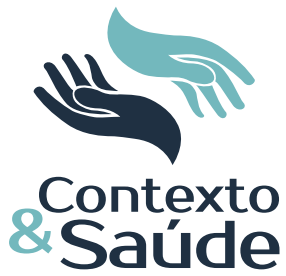

\section{INTRODUÇÃO}

O manejo das complicações consequentes da lesão medular (LM) torna-se fundamental para o bem-estar físico, social e vocacional das pessoas acometidas (AHUJA et al., 2017; SEZER; AKKUS; UĞURLU, 2015). Esse tipo de lesão é caracterizado como uma desordem parcial ou total da funcionalidade da medula espinhal em resposta à interrupção de vias motoras e sensoriais. Essa condição acarreta alterações abaixo do nível da lesão, motoras e sensitivas, superficiais e profundas (WAN; KRASSIOUKOV, 2014).

Dados epidemiológicos demonstram aumento expressivo no número de casos de LM, com destaque para alguns importantes determinantes sociais: jovem, sexo masculino, idade média de 33 anos e ser de país de baixa ou média renda (13,69 por 100.000/pessoas) em comparação com países de alta renda (8,72 por 100.000/pessoas) (KUMAR et al., 2018).

Em pessoas acometidas com LM, a incapacidade funcional pode provocar graus variados de dependência no que se refere às atividades de vida diária (AVDs), principalmente em relação ao autocuidado, controle de esfíncteres, mobilidade, entre outras (TULSKY et al., 2015). A independência funcional influencia o desempenho nas atividades cotidianas, no potencial desempenho ocupacional e na participação social (SLIWINSKI et al., 2020).

Para tanto, na reabilitação é importante que as características de cada indivíduo sejam consideradas no momento de se integrar este indivíduo à tecnologia assistiva (TA), em especial, para esse grupo de pessoas, a cadeira de rodas. A ideia é torná-los mais ativos e funcionais, influenciando, de forma positiva, no alcance funcional, independência e reintegração social (VAN DER SCHEER et al., 2017).

No Brasil, o Comitê de Ajudas Técnicas (CAT) definiu a TA como sendo todo o conjunto de metodologias, recursos e serviços que garantem ou favorecem a ampliação para o desempenho de habilidades funcionais de pessoas com deficiência, promovendo independência, funcionalidade e inclusão social (CAT, 2007).

No presente estudo a TA a ser investigada é a cadeira de rodas. Esta tem tido um papel significativo na rotina de pessoas com LM. Os avanços desta TA, com alternativas mais personalizadas, estende oportunidades para que os indivíduos aumentem sua independência nas AVDs, expandindo a interação com o ambiente e a participação social (OLIVER, 2019).

Tendo as TAs como recursos de uma demanda pessoal de aceitação e como instrumento facilitador para a execução de AVDs pelos indivíduos com LM, é necessária uma melhor compreensão das inter-relações entre a promoção da independência funcional e a satisfação do usuário na utilização deste recurso de TA. Assim, o objetivo deste estudo foi avaliar a correlação entre independência funcional em AVDs e a satisfação com o uso da TA (cadeira de rodas) em pessoas com LM.

\section{MÉTODOS}

Trata-se de um estudo observacional, de corte transversal e analítico. É um tipo de estudo que descreve as características de uma dada população, pos- 
sibilitando a associação entre variáveis. Todas as mensurações foram realizadas em um único momento, como uma fotografia da realidade atual do fenômeno abordado.

A pesquisa foi realizada em um hospital de referência em reabilitação e readaptação na cidade de Goiânia-GO, que é reconhecido pelo Ministério da Saúde como Centro Especializado em Reabilitação por sua atuação na recuperação de pessoas com deficiência física, auditiva, visual e intelectual. É o primeiro hospital público do Centro-Oeste com o certificado de Acreditado com Excelência - Nível 3 - da Organização Nacional de Acreditação (ONA).

O período de realização da pesquisa de campo foi de 15 de julho a 30 de outubro de 2019, utilizando-se de uma amostragem por conveniência. Dessa forma, participaram do estudo 146 indivíduos com LM (tetraplegia e paraplegia). Para a seleção destes participantes da pesquisa foram utilizados os prontuários eletrônicos das pessoas com LM traumática. A busca de dados nos prontuários incluiu o tempo da ocorrência, a causa da lesão, a implicação pós-lesão, a extensão da lesão e a classificação na escala da American Spinal Injury Association (Asia) da LM.

Os critérios de inclusão foram: diagnóstico de lesão medular traumática; maiores de 18 anos; sob atendimento ambulatorial e/ou em academia de reabilitação; clinicamente estáveis; com paraplegia ou tetraplegia e usuários de cadeiras de rodas. Os critérios de exclusão foram: LM não traumática, lesão encefálica associada; pacientes sob regime de internação.

Para alcançar os objetivos do presente estudo foram utilizados três instrumentos de pesquisa. O primeiro foi um questionário sociodemográfico e clínico construído pelos pesquisadores, que visou à identificação das variáveis da vida dos indivíduos.

O segundo foi a Spinal Cord Independence Measure - Self Reported Version (brSCIM-SR). Este é um instrumento específico para pessoas acometidas de LM. É uma escala de medida da independência funcional em LM em autorrelato, composto de 17 tarefas de AVDs relevantes às pessoas com LM. A versão de autorrelato Spinal Cord Independence Measure - Self-Reported (SCIM-SR) foi desenvolvida na Suíça a partir da versão do Spinal Cord Independence Measure - III (SCIM-III). Esse instrumento objetiva avaliar e mensurar a funcionalidade de pessoas com LM em suas AVDs. A tradução, adaptação cultural e validação da brSCIM-SR para o idioma português do Brasil foi publicada por llha et al. (2016).

Este instrumento é agrupado em três subseções: a) autocuidado (escore varia de zero a 20); b) respiração e controle de esfíncteres (escore varia de zero a 40); e c) mobilidade (escore varia de zero a 40). Sua pontuação total (escore geral) varia de zero a cem. Quanto mais elevado o escore, maiores são os níveis de independência funcional (ILHA et al., 2016).

O terceiro foi o Quebec User Evaluation of Satisfaction with Assistive Technology (QUEST 2.0). É uma escala que foi originalmente desenvolvida nos idiomas inglês e francês. A tradução, adaptação cultural e validação do QUEST 2.0 para o idioma português do Brasil foi realizada por Carvalho, Gois Júnior e Sá (2014). Esta tem por objetivo medir a satisfação de usuários de tecnologias assis- 
tivas em diversos aspectos, justificando a necessidade do uso efetivo destes dispositivos. Contém 12 itens com uma escala que varia de zero a 5 para mensurar o grau de satisfação, sendo determinado em: insatisfeito, pouco satisfeito, mais ou menos satisfeito, bastante satisfeito, totalmente satisfeito.

O primeiro estágio, denominado de "recursos de TA", consiste em oito itens relacionados ao uso das tecnologias assistivas (dimensões, peso, ajustes, segurança, durabilidade, facilidade de uso, conforto e eficácia). O segundo estágio, denominado de "serviços", consiste de quatro itens relacionados à prestação de serviço (processo de entrega, reparos e assistência técnica, serviços profissionais e acompanhamento) (CARVALHO; GOIS JÚNIOR; SÁ, 2014). No presente estudo o instrumento foi empregado para avaliar o grau de satisfação apenas em relação à cadeira de rodas.

O escore geral da escala é obtido pela soma simples dos 12 itens divididos pelo número de itens (média). Os escores, geral e das dimensões, variam de 1,00 a 5,00. Quanto maior o escore maior é o nível de satisfação com a TA (CARVALHO; GOIS JÚNIOR; SÁ, 2014).

Com os dados coletados foi confeccionado um banco de dados utilizando o software IBM SPSS Statistics23. Posteriormente foi realizada estatística descritiva com o cálculo medidas de tendência central para as variáveis contínuas e cálculo das frequências absoluta e relativa percentual para as variáveis discretas.

Na sequência foi aplicado teste de normalidade (Kolmogorov-Smirnov) para distinguir as distribuições paramétricas e não paramétricas, com o intuito de comparação dos resultados do questionário estratificado pelas variáveis sociodemográficas. Foram utilizados, para as distribuições paramétricas, os testes t de Student e Anova, e para as distribuições não paramétricas os testes Mann-Whitney e Kruskal-Wallis. Para verificar a correlação da independência funcional nas AVDs com a satisfação do uso da TA, foi utilizado o teste de correlação de Pearson. Para todos os testes comparativos foi assumido $p$-valor menor ou igual a 0,05 como significativo.

Antes de iniciar a coleta de dados, o presente trabalho foi encaminhado ao Comitê de Ética e Pesquisa (CEP) da Pontifícia Universidade Católica de Goiás (PUC Goiás), quando foi aprovado com o parecer n. 3.425.341. Todos os participantes desta pesquisa leram e assinaram o Termo de Consentimento Livre e Esclarecido (TCLE).

\section{RESULTADOS}

A amostra foi composta por 146 indivíduos com LM traumática. Em relação ao perfil sociodemográfico, a idade variou de18 a 69 anos, com média de $37,8( \pm 12,6)$. A maioria era do sexo masculino $(82,9 \%)$, solteiro $(52,7 \%)$, com nível médio de escolaridade $(53,4 \%)$, aposentados $(52,7 \%)$ ou recebendo auxílio doença $(30,8 \%)$ do Instituto Nacional de Seguridade Social (INSS) (Tabela 1). 
Tabela 1 - Dados sociodemográficos dos indivíduos com lesão medular traumática, Goiânia, Goiás, Brasil, 2020

\begin{tabular}{|c|c|c|}
\hline Variáveis ( $n=146$ ) & $\mathbf{N}$ & (\%) \\
\hline \multicolumn{3}{|l|}{ Idade (anos) } \\
\hline Até 35 & 67 & 45,9 \\
\hline Acima de 35 & 79 & 54,1 \\
\hline \multicolumn{3}{|l|}{ Sexo } \\
\hline Masculino & 121 & 82,9 \\
\hline Feminino & 25 & 17,1 \\
\hline \multicolumn{3}{|l|}{ Estado civil } \\
\hline Solteiro & 77 & 52,7 \\
\hline Casado/União estável & 49 & 33,6 \\
\hline Divorciado/Separado & 16 & 11,0 \\
\hline Viúvo & 4 & 2,7 \\
\hline \multicolumn{3}{|l|}{ Filhos } \\
\hline Sim & 73 & 50,0 \\
\hline Não & 73 & 50,0 \\
\hline \multicolumn{3}{|l|}{ Escolaridade } \\
\hline Analfabeto & 3 & 2,1 \\
\hline Ensino Fundamental & 38 & 26,0 \\
\hline Ensino Médio & 78 & 53,4 \\
\hline Ensino Superior & 27 & 18,5 \\
\hline \multicolumn{3}{|l|}{ Renda mensal } \\
\hline Sem renda & 12 & 8,2 \\
\hline Até 1 salário mínimo & 80 & 54,8 \\
\hline Entre 1 a 5 salário mínimo & 51 & 34,9 \\
\hline acima de 5 salário mínimo & 3 & 2,1 \\
\hline \multicolumn{3}{|l|}{ Maior fonte de renda } \\
\hline Aposentadoria & 77 & 52,7 \\
\hline Auxílio doença & 45 & 30,8 \\
\hline Trabalho & 10 & 6,8 \\
\hline Outras & 2 & 1,4 \\
\hline Sem renda & 12 & 8,2 \\
\hline \multicolumn{3}{|c|}{ Trabalho remunerado antes da LM } \\
\hline Sim & 123 & 84,2 \\
\hline Não & 23 & 15,8 \\
\hline
\end{tabular}

Fonte: Elaborada pelos autores, 2020.

Dentre as características clínicas da LM, o maior fator causal foi o acidente de trânsito (50\%), seguido de arma de fogo $(22,6 \%)$, quando apresentaram lesão completa $(58,9 \%)$ resultando em paraplegia $(65,1 \%)$. O nível motor mais frequente foi de T7-T12 (32,9\%). A maioria dos participantes apresentou histórico de ocorrência da LM acima de três anos (72,6\%) (Tabela 2). 
Tabela 2 - Caracterização dos aspectos clínicos dos indivíduos com lesão medular traumática, Goiânia, Goiás, Brasil, 2020

\begin{tabular}{lcc}
\hline Variáveis ( $\mathbf{n}$ - 146) & N & (\%) \\
\hline Causa da lesão & & \\
Acidente de trânsito & 73 & 50,0 \\
Arma de fogo & 33 & 22,6 \\
Acidente de trabalho & 25 & 17,1 \\
Mergulho em águas rasas & 9 & 6,2 \\
Acidente esportivo & 3 & 2,1 \\
Arma branca & 3 & 2,1 \\
Tempo de lesão & & \\
Até 1 ano & 14 & 9,6 \\
Entre 1 e 3 anos & 26 & 17,8 \\
Acima de 3 anos & 106 & 72,6 \\
Implicação pós-lesão & & \\
Paraplegia & 95 & 65,1 \\
Tetraplegia & 51 & 34,9 \\
Extensão da lesão & & \\
Completa & 86 & 58,9 \\
Incompleta & 60 & 41,1 \\
Classificação Asia & & \\
A & 86 & 58,9 \\
B & 22 & 15,1 \\
C & 30 & 20,5 \\
D & 8 & 5,5 \\
Nível motor & & \\
C2-C7 & 51 & 34,9 \\
T1-T6 & 37 & \\
T7-T12 & 48 & \\
L1-L5 & 10 & \\
\hline
\end{tabular}

Fonte: Elaborada pelos autores, 2020.

$\mathrm{Na}$ avaliação da independência funcional em AVDs, identificou-se uma média no domínio respiração e esfíncteres de $26,4( \pm 7,4)$, na mobilidade de $14,3( \pm 5,5)$ e no autocuidado de $14,6( \pm 5,3)$. No escore geral a média foi de $55,3( \pm 15,8)$ (Tabela 3$)$.

Tabela 3 - Caracterização da medida de independência funcional (brSCIM-SR) dos indivíduos com lesão medular traumática, Goiânia, Goiás, Brasil, 2020

\begin{tabular}{lcccc}
\hline Variáveis (n = 146) & Média & DP & Mínimo & Máximo \\
\hline Autocuidado (zero a 20) & 14,6 & 5,3 & 0 & 20 \\
Respiração/Esfíncteres(zero a 40) & 26,4 & 7,4 & 10 & 40 \\
Mobilidade (zero a 40) & 14,3 & 5,5 & 0 & 20 \\
\hline Escore Geral (zero a 100) & $\mathbf{5 5 , 3}$ & $\mathbf{1 5 , 8}$ & $\mathbf{1 6}$ & $\mathbf{7 9}$ \\
\hline
\end{tabular}

Fonte: Elaborada pelos autores, 2020. 
Na avaliação de satisfação com a TA (cadeira de rodas) verificou-se um maior escore médio no domínio da satisfação com o recurso $3,86( \pm 0,74)$. Já no domínio da prestação de serviços o escore médio foi de $3,21( \pm 1,18)$ e o escore geral de satisfação com a cadeira de rodas foi 3,64 $( \pm 0,77)$ (Tabela 4).

Tabela 4 - Caracterização dos níveis de satisfação com a TA (QUEST 2.0) dos indivíduos com lesão medular traumática, Goiânia, Goiás, Brasil, 2020

\begin{tabular}{lcccc}
\hline Variáveis $(\mathbf{n}=\mathbf{1 4 6})$ & Média & DP & Mínimo & Máximo \\
\hline Recursos de TA (1,00 a 5,00) & 3,86 & 0,74 & 1,50 & 5,00 \\
Serviços (1,00 a 5,00) & 3,21 & 1,18 & 1,00 & 5,00 \\
\hline Escore Geral (1,00 a $\mathbf{5 , 0 0})$ & $\mathbf{3 , 6 4}$ & $\mathbf{0 , 7 7}$ & $\mathbf{1 , 3 3}$ & $\mathbf{5 , 0 0}$ \\
\hline
\end{tabular}

Fonte: Elaborada pelos autores, 2020.

A análise de correlação de Pearson não revelou associação entre a independência funcional nas AVDs e a satisfação com a cadeira de rodas (Tabela 5).

Tabela 5 - Análise de correlação de Pearson entre a medida de independência funcional (brSCIM-SR) e os níveis de satisfação com a TA (QUEST 2.0) dos indivíduos com lesão medular traumática, Goiânia, Goiás, Brasil, 2020

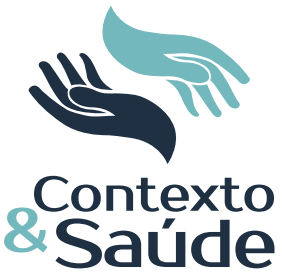

Correlação

Escore geral dos níveis de atividade da vida diária de Pearson

$x$ Escore geral dos níveis de satisfação com a cadeira de rodas

$\begin{array}{ll}r \text { (Pearson) } & -0,0963 \\ p \text {-Valor } & 0,2477\end{array}$

Fonte: Elaborada pelos autores, 2020

\section{DISCUSSÃO}

No presente estudo o perfil da amostra foi predominantemente composto por homens solteiros em idade produtiva, com Ensino Médio, aposentados por invalidez ou recebendo auxílio doença da previdência pública. A paraplegia completa predominou, e as principais causas foram acidente de trânsito e arma de fogo.

É um perfil que se assemelha aos dados publicados por uma metanálise da região do Oriente Médio e Norte da África, que incluiu sete países (Turquia, Irã, Arábia Saudita, Egito, Jordânia, Kuwait e Catar). Foi identificado um perfil como sendo do sexo masculino, com média de idade de 31, 32 anos, com paraplegia completa e predomínio de lesões em nível torácico. A faixa etária mais comumente afetada foi de 20 a 29 anos e depois de 30 a 39 anos. Os acidentes automotores foram a principal causa, seguidos de quedas, arma de fogo, violência e esportes (ELSHAHIDI et al., 2018).

No México, dois estudos também apresentaram dados próximos aos evidenciados no presente estudo, com predomínio de homens com idade média de 37,9 anos, solteiros e com baixa escolaridade. Há uma diferença em relação 
à causa da lesão, sendo a mais comum a queda de altura superior a um metro seguida de acidentes com veículos automotores e violência. A maioria das lesões também resultou em paraplegia completa (ALMENDÁREZ-SÁNCHES, 2020; RODRÍGUEZ-MEZA et al., 2016).

Dados dos Estados Unidos da América indicam que a média de idade na lesão aumentou de 28,7 anos na década de 70 do século 20 para 42,2 anos de 2010 a 2014. Os níveis médios de educação e a porcentagem de solteiros aumentaram. As colisões veiculares continuam a ser a principal causa, e as lesões resultantes de quedas aumentaram particularmente entre os 46 anos. Aumento progressivo de lesões cervicais altas e incompletas foi observado para vários grupos de idade e etiologia (CHEN; HE; DEVIVO, 2016).

A tendência de mudança da epidemiologia da lesão medular traumática diz respeito ao aumento gradual da idade média das pessoas, uma proporção maior de lesões relacionadas a quedas da própria altura e aumento das lesões cervicais e incompletas, especialmente entre os mais idosos (BÁRBARA-BATALLER et al., 2018; KUDO et al., 2019; MIRZAEVA et al., 2019; MITCHELL et al., 2020; SMITH et al., 2019). Estes estudos sugerem a necessidade de uma avaliação de risco multidimensional e intervenção multifatorial, especialmente para reduzir quedas e lesão medular em idosos.

As diferenças epidemiológicas da lesão medular entre países e regiões são resultantes das características populacionais, geográficas, condições socioeconômicas, científicas e tecnológicas. Torna-se necessária uma maior compreensão da epidemiologia para estabelecer medidas de intervenção e prevenção apropriadas, adaptadas às tendências regionais de acordo com as particularidades de cada população.

Após o acometimento de uma LM, o indivíduo passa por um período exaustivo de hospitalização; isso significa um longo tempo para entender o impacto resultante de sua nova condição. É no processo de reabilitação que a pessoa irá aprender as habilidades necessárias para sua reintegração na comunidade. Um aspecto particularmente importante nesse processo é o retorno ao trabalho.

Os participantes deste estudo apresentaram predomínio de nível médio de escolaridade. Embora nem todos os estudos epidemiológicos descrevam esse dado, isso é importante, por se tratar de pessoas jovens, em pleno desenvolvimento profissional e idade produtiva, o que corrobora os outros estudos (AHMED et al., 2018; ALMENDÁREZ-SÁNCHEZ, 2020; RODRÍGUEZ-MEZA et al., 2016).

A escolaridade pode ser fator relevante e determinante do retorno ao trabalho para indivíduos com lesão medular. Um estudo realizado em Bangladesh identificou que $44 \%$ dos participantes com lesão medular estavam desempregados e $65 \%$ viviam abaixo da linha da pobreza, com qualidade de vida e participação reduzidas (HOSSAIN et al., 2019).

Para as pessoas vítimas de LM, a importância do trabalho está ligada a conexões sociais, identidade, participação e integração na social, senso de valor, economia e as rotinas do cotidiano (LEIULFSRUD, 2016). Pessoas que não têm Ensino Superior ou não têm emprego viável para retornar após a lesão, parecem 
estar vulneráveis no retorno ao trabalho (HOLMLUND et al., 2018). Indivíduos mais jovens, com maior nível de escolaridade, maior independência funcional e suporte social, estão associados a maiores oportunidades de trabalho, integração na comunidade e maior satisfação com a vida (AHMED et al., 2018; HUANG, 2017).

Em se tratando da independência funcional em AVDs, a média total foi de 55,3 . Um programa de reabilitação vocacional interdisciplinar, em uma comunidade de Singapura, avaliou a independência funcional antes e após a reabilitação física utilizando a escala SCIM-III. Antes de iniciar o programa a pontuação total foi 55 e média maior no domínio respiração e esfíncteres, corroborando achados do presente estudo (KEE et al., 2020).

Identifica-se que indivíduos com LM traumática, quando comparados aos com lesão não traumática, apresentam mais limitações. A LM de nível mais alto e mais grave, com base na Asia, tem associação mais forte com mais limitações de atividade funcionais. Assim, é de grande relevância as características da lesão para o desempenho das AVDs (JORGENSEN; IWARSSON; LEXELL, 2017).

$\mathrm{Na}$ avaliação de satisfação com a TA verificou-se que o nível de satisfação com a cadeira de rodas atingiu uma média maior que a prestação de serviço. Estes resultados estão alinhados com os dados apresentados por um estudo realizado na Tanzânia, quando os participantes ficaram satisfeitos com as características do recurso de TA, mas não com a prestação de serviços (AMOSUN; NDOSI; BUCHANAN, 2016). A TA impactou de forma positiva as necessidades de atividade e participação social (AMOSUN; NDOSI; BUCHANAN, 2016).

No presente estudo não houve correlação significativa entre a medida de independência funcional e a satisfação com a TA. Um estudo coreano corrobora tais resultados na medida em que também não foi encontrada tal associação significativa entre a versão coreana do QUEST-K com a SCIM-III e o índice de Barthel modificado (HWANG; HWANG; CHUNG, 2015).

Em contraste com os resultados do estudo, uma pesquisa que utilizou um instrumento de avaliação da mobilidade funcional (FMA) encontrou correlação significativa na satisfação do usuário com o dispositivo de mobilidade. Na amostra de 47 participantes, entretanto, foram incluídas pessoas com diversos diagnósticos, como LM, osteoartrite da coluna vertebral e joelho, mielomeningocele, síndromes genéticas não especificadas, poliomielite, paralisia cerebral, amputação de membros inferiores e tumor na coluna vertebral (PAULISSO et al., 2019).

\section{CONCLUSÃO}

No presente estudo identificou-se que os indivíduos com LM apresentaram um resultado mediano no escore geral da independência funcional. Em relação à medida de independência funcional, a subescala de autocuidado apresentou melhor desempenho entre os três domínios. Além disso altos níveis de satisfação com a cadeira de rodas foram identificados, de forma específica mais com o recurso de TA do que com a prestação de serviços. 
Não foi encontrada, neste estudo, correlação entre a independência funcional e a satisfação do uso de TA. Esse achado pode estar relacionado às próprias limitações do estudo, em que a satisfação com a TA pode ter sido inspirada pela heterogeneidade de fatores que podem influenciar no uso das cadeiras de rodas e sua relação subjetiva com o usuário. Além disso, as diferenças na classificação e níveis de lesão podem refletir na média do escore total da medida de independência funcional, posto que a funcionalidade está diretamente relacionada com a gravidade e o grau da lesão.

Ademais, o presente estudo viabiliza novas evidências que contribuem para o conhecimento acerca da relação entre independência funcional e a satisfação das pessoas com LM com a cadeira de rodas. Torna-se necessário, portanto, o desenvolvimento de estratégias multidisciplinares que promovam e incentivem a reabilitação de indivíduos com LM a fim de favorecer uma reinserção integral à sociedade. Para tanto, faz-se necessário o entendimento existente na relação entre a independência funcional e a satisfação com o uso do recuso de TA.

\section{REFERÊNCIAS}

AHMED, N. et al. Community integration and life satisfaction among individuals with spinal cord injury living in the community after receiving institutional care in Bangladesh. Disability and Rehabilitation, v. 40, n. 9, p. 1.033-1.040, 2018.

AHUJA, C. S. et al. Traumatic spinal cord injury. Nature Reviews Disease Primers, v. 3, n. 17.018, p. 1-21, 2017.

ALMENDÁREZ-SÁNCHEZ, C. A. et al. Technical note: in Mexico, the majority of 147 traumatic spinal cord injuries occurred in the thoracic spine for young males. Surgical Neurology International, v. 11, n. 162, p. 1-6, 2020.

AMOSUN, S.; NDOSI, A.; BUCHANAN, H. Locally manufactured wheelchairs in Tanzaniaare users satisfied? African Health Sciences, v. 16, n. 4, p. 1.174-1.181, 2016.

BÁRBARA-BATALLER, E. et al. Change in the profile of traumatic spinal cord injury over 15 years in Spain. Scandinavian Journal of Trauma, Resuscitation and Emergency Medicine, v. 26, n. 1, p. 1-8, 2018.

CARVALHO, K. E. C.; GOIS JÚNIOR, M. B.; SÁ, K. N. Tradução e validação do Quebec user evaluation of satisfaction with assistive technology (QUEST 2.0) para o idioma português do Brasil. Revista Brasileira de Reumatologia, v. 54, n. 4, p. 260-267, 2014.

CAT. Comitê de Ajudas Técnicas. Ata da Reunião VII, 2007. 2007. Disponível em: http:// www.comunicacaoalternativa.com.br/artigoscientificos/\%0AAta_VII_Reunião_do_Comi te_de_Ajudas_Técnicas.\%0Apdf?attredirects=0\&d=1. Acesso em: 14 abr. 2019.

CHEN, Y.; HE, Y.; DEVIVO, M. J. Changing demographics and injury profile of new traumatic spinal cord injuries in the United States,1972-2014. Archives of Physical Medicine and Rehabilitation, v. 97, n. 10, p. 1.610-1.619, 2016.

ELSHAHIDI, M. H. et al. Epidemiological Characteristics of Traumatic Spinal Cord Injury (TSCl) in the Middle-East and North-Africa (MENA) Region: a systematic review and meta-analysis. Bulletin of Emergency and Trauma, v. 6, n. 2, p. 75-89, 2018.

HOLMLUND, L. et al. Return to work in the context of everyday life $7-11$ years after spinal cord injury - a follow-up study. Disability and Rehabilitation, v. 40, n. 24, p. 2.875 2.883, 2018.

HOSSAIN, M. S. et al. Health status, quality of life and socioeconomic situation of people with spinal cord injuries six years after discharge from a hospital in Bangladesh. Spinal Cord, v. 57, n. 8, p. 652-661, 2019.

HUANG, I. C. Employment outcomes following spinal cord injury in Taiwan. International Journal of Rehabilitation Research, v. 40 n. 1, p. 84-90, 2017. 
HWANG, W. J.; HWANG, S.; CHUNG, Y. Test-retest reliability of the Quebec user evaluation of satisfaction with assistive technology 2.0-Korean version for individuals with spinal cord injury. The Journal of Physical Therapy Science, v. 27, n. 5, p. 1.291-1.293, 2015. ILHA, J. et al. Tradução e adaptação transcultural da versão brasileira da Spinal Cord Independence Measure - Self - Reported Version (brSCIM-SR). Revista Brasileira de Neurologia, v. 52, n. 1, p. 2-17, 2016

JORGENSEN, S.; IWARSSON, S.; LEXELL, J. Secondary health conditions, activity limitations, and life satisfaction in older adults with long-term spinal cord injury. $P M \& R$ : The Journal ff Injury, Function and Rehabilitation, v. 9, n. 4, p. 356-366, 2017.

KEE, K. M. et al. Return to work after spinal cord injury: a Singaporean pilot community-based rehabilitation program. Spinal Cord, v. 58, n. 10, p. 1.096-1.103, 2020.

KUDO, D . et al. An epidemiological study of traumatic spinal cord injuries in the fastest aging area in Japan. Spinal Cord, v. 57, n. 6, p. 509-515, 2019.

KUMAR, R. et al. Traumatic spinal injury: global epidemiology and worldwide volume. World Neurosurgery, v. 113, p. e345-e363, 2018.

LEIULFSRUD, A. S. et al. The meaning of employment from the perspective of persons with spinal cord injuries in six European countries. Work, v. 55, n.1, p. 133-144, 2016

MIRZAEVA, L. et al. Incidence of adult traumatic spinal cord injury in Saint Petersburg, Russia. Spinal Cord, v. 57, n. 8, p. 692-699, 2019.

MITCHELL, J. et al. Epidemiology of traumatic spinal cord injury in New Zealand (20072016). New Zealand Medical Journal, v. 133, n. 1.509, p. 47-57, 2020.

OLIVER, M. Assistive technology in polytrauma rehabilitation. Physical Medicine and Rehabilitation Clinical North America, v. 30, n. 1, p. 217-259, 2019.

PAULISSO, D. C. et al. Functional mobility assessment is reliable and correlated with satisfaction, independence and skills. Assistive Technology, p. 1-7, 2019. doi: 10.1080/10400435.2019.1629125.

RODRÍGUEZ-MEZA, M. V. et al. Clinical and demographic profile of traumatic spinal cord injury: a Mexican hospital-based study. Spinal Cord, v. 54, n. 4, p. 266-269, 2016.

SEZER, N.; AKKUS, S.; UĞURLU, F, G. Chronic complications of spinal cord injury. World Journal of Orthopedics, v. 6, n. 1, p. 24-33, 2015.

SLIWINSKI, M. M. et al. Community exercise programing and its potential influence on quality of life and functional reach for individuals with spinal cord injury. The Journal of Spinal Cord Medicine, v. 43, n. 3, p. 358-363, 2020.

SMITH, E. et al. Prospective epidemiological update on traumatic spinal cord injury in Ireland. Spinal Cord Series and Cases, v. 5, p. 1-9, 2019.

TULSKY, D. S. et al. Overview of the spinal cord injury - quality of life (SCI-QOL) measurement system. The Journal of Spinal Cord Medicine, v. 38, n. 3, p. 257-269, 2015.

VAN DER SCHEER, J. W. et al. Effects of exercise on fitness and health of adults with spinal cord injury: a systematic review. Neurology, v. 89, n. 7, p. 736-745, 2017.

WAN, D.; KRASSIOUKOV, A. V. Life-threatening outcomes associated with autonomic dysreflexia: A clinical review. The Journal of Spinal Cord Medicine, v. 37, n. 1, p. 2-10, 2014. 